\title{
ARTICLE \\ Factor VII deficiency: a novel missense variant and genotype- phenotype correlation in patients from Southern Italy
}

\author{
Giovanni Tiscia ${ }^{1}$, Giovanni Favuzzi ${ }^{1}$, Elena Chinni ${ }^{1}$, Donatella Colaizzo ${ }^{1}$, Lucia Fischetti ${ }^{1}$, Mariano Intrieri ${ }^{2}$, Maurizio Margaglione ${ }^{3}$ and
} Elvira Grandone ${ }^{1}$

This study aimed at attempting to correlate genotype and phenotype in factor VII deficiency. Here, we present molecular and clinical findings of 10 patients with factor VII deficiency. From 2013 to 2016, 10 subjects were referred to our center because of a prolonged prothrombin time identified during routine or presurgery examinations or after a laboratory assessment of a bleeding episode. Mutation characterization was performed using the bioinformatics applications PROMO, SIFT, and Polyphen-2. Structural changes in the factor VII protein were analyzed using the SPDB viewer tool. Of the 10 variants we identified, 1 was responsible for a novel missense change (c.1199G >C, p.Cys400Ser); in 2 cases we identified the c.-54G >A and c.509G >A (p.Arg170His) polymorphic variants in the $5^{\prime}$-upstream region of the factor VII gene and exon 6 , respectively. To our knowledge, neither of these polymorphic variants has been described previously in factor VII-deficient patients. In silico predictions showed differences in binding sites for transcription factors caused by the c.-54G $>$ A variant and a probable damaging effect of the p.Cys400Ser missense change on factor VII active conformation, leading to breaking of the Cys400-Cys428 disulfide bridge. Our findings further suggest that, independently of factor VII levels and of variants potentially affecting factor VII levels, environmental factors, e.g., trauma, could heavily influence the clinical phenotype of factor VII-deficient patients.

Human Genome Variation (2017) 4, 17048; doi:10.1038/hgv.2017.48; published online 2 November 2017

\section{INTRODUCTION}

Blood coagulation consists of three imbricated phases, 'initiation', 'amplification', and 'propagation', that culminate in the formation of a fibrin clot. ' Factor VII (FVII) is involved in the 'initiation' phase and acts by binding tissue factor (TF) exposed by cells after endothelial injury. ${ }^{2}$ The activated FVII (FVIla)/TF complex promotes activation of factor $X(F X)$ and factor IX (FIX), leading to the generation of a small quantity of thrombin. ${ }^{3} \mathrm{FVII}$ is synthesized by hepatocytes and secreted into the blood as a single chain molecule of $\sim 50000$ daltons. ${ }^{4}$ Hydrolysis of the Arg152-lle153 bond determines activation of FVII. $^{5}$ The resulting FVIla is processed into a light chain (152 residues) and a heavy chain (254 residues), joined to each other by a disulfide bridge. ${ }^{5}$ The FVIla structure is organized in a gamma-carboxyglutamic acid-rich motif (Gla motif), two epidermal growth factor (EGF)-like motifs, and a C-terminal serine-protease motif (SP-motif). The FVII gene (F7) maps to chromosome 13 (13q34), stretches along $12.8 \mathrm{~kb}$ and contains 9 exons. $^{6}$

Inherited FVII deficiency is an autosomal recessive trait and constitutes the most frequently diagnosed rare coagulation disorder, with an observed incidence of between 1:300 000 and 1:500 000 . $^{7}$ FVII deficiency is defined by the absence or levels below $70 \%$ of normal of this clotting factor. ${ }^{8}$

Several variants have been identified in $F 7$, mostly in exon 9 (coding for the SP-motif) and as variants responsible for missense changes. ${ }^{9}$ However, other variants have been found in other F7 regions; these can also be nonsense and insertion/deletions and may influence the splicing process. In addition, a series of polymorphic variants that could modulate FVII levels are frequently found in FVII-deficient subjects. ${ }^{10}$
The FVII deficiency has no peculiarities with regard to clinical manifestations, which can be easy bruising, hematomas, epistaxis, gastrointestinal bleeding, hematuria, and vaginal bleeding. Vaginal bleeding and iron deficiency are commonly found in women with the FVII deficiency. ${ }^{7}$ Hemarthroses are uncommon manifestations of the FVII deficiency, while intracranial hemorrhages could be fatal manifestations of the FVII deficiency. ${ }^{11}$

It is a challenge to evaluate the bleeding risk based on FVII levels in FVII deficiency. ${ }^{12}$ Homozygotes and compound heterozygotes show very low FVII levels, but they can be asymptomatic or show a bleeding diathesis with higher FVII levels. ${ }^{13}$ Heterozygotes can be asymptomatic or mildly symptomatic; however, data available on these individuals are limited. ${ }^{13}$ This study aimed at correlating genotype and phenotype in FVII deficiency. Here we present molecular and clinical findings from a cohort of 10 subjects with FVII deficiency including also description of the heterozygotes; one novel missense change and two previously undescribed polymorphic variants have been found.

\section{MATERIALS AND METHODS}

Patients

From 2013 to 2017, 10 individuals from southern Italy were referred to our center because of a prolonged prothrombin time (PT) identified during routine or presurgery examinations or during laboratory assessment of a bleeding episode. Clinical history was collected in a specific questionnaire focusing on any bleeding episodes. Attention was paid to epistaxis, vaginal bleeding, hematomas, hemarthrosis, gastrointestinal tract or intracranial hemorrhages and to bleeding outcomes of hemostatic challenges with/ without replacement therapy. To fully assess the presence of other possible causes predisposing to a bleeding tendency, a blood cell count

\footnotetext{
${ }^{1}$ Atherosclerosis and Thrombosis Unit, I.R.C.C.S. 'Casa Sollievo della Sofferenza', San Giovanni Rotondo (Foggia), Italy; ${ }^{2}$ Department of Medicine and Health Sciences 'Vincenzo Tiberio', University of Molise, Campobasso, Italy and ${ }^{3}$ Medical Genetics, University of Foggia, Foggia, Italy.

Correspondence: E Grandone (e.grandone@operapadrepio.it)

Received 18 May 2017; revised 23 August 2017; accepted 10 September 2017
} 
was also done in addition to clotting screening performed during the bleeding episodes, in order to exclude thrombocytopenia. The presence of vitamin $\mathrm{K}$ deficiency, due to diet or warfarin, was also excluded as another cause of prolonged PT, such as abnormal liver function. Acquired FVII deficiency, due to malignancy, aplastic anemia, medicaments or stem cell transplantation, was excluded. Informed consent was provided by all patients to permit use of their personal clinical and molecular information. The study complied with the Declaration of Helsinki Principles and was approved by the ethics committee of our institution.

\section{Plasma- and DNA-based investigations}

Plasma- and DNA-based investigations were performed on samples obtained from peripheral whole blood. Blood specimens were collected in BD Vacutainer Citrate Tubes with $3.8 \%$ buffered sodium citrate. The plasma samples were prepared by a centrifugation procedure at $3000 \mathrm{~g}$ for 10 min and stored at $-80^{\circ} \mathrm{C}$ until use. The FVII activity assay (FVII:C) was performed by a one-stage method on a BCS System (Siemens Healthcare Diagnostic Products GMbH, Marburg/Germany). DNA was extracted according to standard protocol. ${ }^{14}$ Research of possible variants of $F 7$ was carried out by amplifying $5^{\prime}$-upstream regions, coding sequences and exon/intron boundaries. Amplification reactions were achieved by using sense and anti-sense oligonucleotides designed and numbered on the basis of known sequences of $F 7$ (NCBI Reference Sequence number NM_001267554). Amplified fragments were subjected to direct cycle sequencing using a BigDye Terminator v3.1 Cycle Sequencing kit and the ABI PRISM 3130 Genetic Analyzer Sequencer (PE Biosystems, Foster City, (A, USA).

\section{In silico predictions and molecular modeling}

To investigate possible effects due to the presence of a variant identified in regulatory regions of $F 7$, predictions of potential binding sites for transcription factors in DNA sequences of $F 7$ were made. Thus, the weight matrix-based virtual laboratory PROMO ${ }^{15}$ implemented by the TRANSFAC 8.3 (dissimilarity margin $\leqslant 15 \%$ ) application was used to verify whether a variant could hypothetically influence transcription factor binding. A fragment spanning nucleotides -76 to -32 of the $F 75^{\prime}$-UTR region, including wild-type and mutant alleles, was used as the target sequence in the PROMO program. Comparative genomic analysis was performed by multiple alignment within Ensembl computational infrastructure in order to evaluate conservation rate at position c.-54 across eight eutherian mammalian species. ${ }^{16}$ A graphical representation of the comparative genomic analysis was obtained by the WebLogo application (http:// weblogo.berkeley.edu/logo.cgi): the overall height of the stack indicates the sequence conservation at that position, while the height of the symbols within the stack indicates the relative frequency of the nucleic acid at that position. ${ }^{17}$ To verify whether missense changes involve critical residues in FVII protein, multiple alignments of FVII sequences were generated using the computer program MUSCLE (version 3.6$)^{18}$ on the HomoloGene automated system (http://www.ncbi.nlm.nih.gov/homolo gene). Deleterious and damaging effects of missense changes were predicted using the web-based tools SIFT (Sorting Intolerant from Tolerant, http://sift.bii.a-star.edu.sg/) ${ }^{19}$ and Polyphen-2 (Polymorphism Phenotyping v2, http://genetics.bwh.harvard.edu/pph2/). ${ }^{20}$ Residue changes were classified as deleterious with SIFT prediction scores 0.05 and a Median Conservation Information value $<3.25$ and damaging with Polyphen-2 predictions according to the score ranging $0-1$, with a score of 1 indicating a probable damaging residue Finally, to model and visualize the FVII structure (PDB code: 2C4F) in the presence of wild-type and mutant residues, the Swiss PdbViewer (v4.1) ${ }^{21}$ application was used.

\section{RESULTS}

All patients were diagnosed with a FVII deficiency in the presence of a prolonged PT, corrected by normal plasma, combined with a normal activated partial thromboplastin time (aPTT) and fibrinogen. In the presence of a prolonged PT, possible acquired conditions, e.g., vitamin $\mathrm{K}$ deficiency, ongoing warfarin treatment, abnormal liver function, were excluded. As summarized (Table 1), we identified 1 homozygous, 2 compound heterozygous and 7 heterozygous individuals. Among the 7 heterozygous individuals, 1 (\#2) showed a novel missense change in exon 9. In addition, we identified two undescribed polymorphic variants,

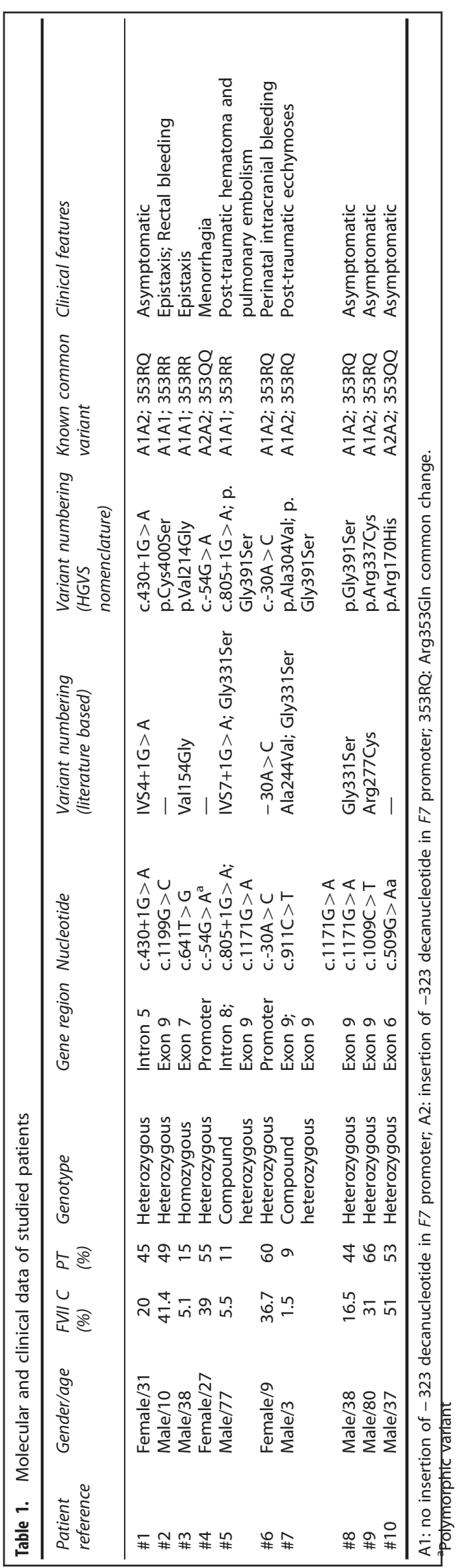


a

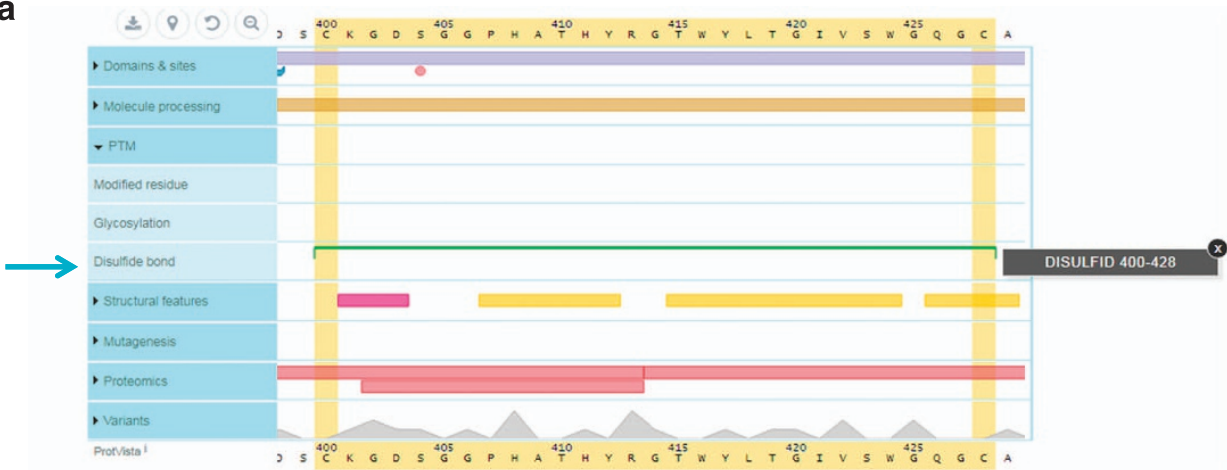

b

H.sapiens IRQSRKVGDSPNTEYMFCAGYSDGSKDSCRGDSGGPHATHYRGTWYITG P.troglodytes IRQSRKVGDSPNITEYMECAGYSDGSKDS CKGDSGGPHATHYRGTWYITG

M.mulatta

C.lupus

B.taurus IQQSQRAEASPNITEYMFCAGYSDGSRDS CKGDSGGPHATRYRGTWYITG QERSRRRSGSPAITENMFCAGYIDGSKDA C RGDSGGPHATKFQGTWYITG IQQSRQRPGGPVVTDNMFCAGYSDGSKDA CKGDSGGPHATRERGTWFITG M.musculus IEHAKHSSNTPKITENMFCAGYMDGTKDZCKGDSGGPHATHYHGTWYITG R.norvegicus IEHAKHSANTPRITENMFCAGYMDGTKDZC CGDSGGPHATHYHGTWYITG G.gallus EKQANI-----NITENMFCAGDITGKKDSCKGDSGGPHATKYKNTWFITG D.rio RARSGL-----TVSRNMICAGEAEGGRDSCRGDSGGPIVTRYRNTWFITG

$X$.tropicalis IRQTQM-----NISRNMFCAGYTDGSKDSC RGDSGGPHATQYKNTHFITG

C
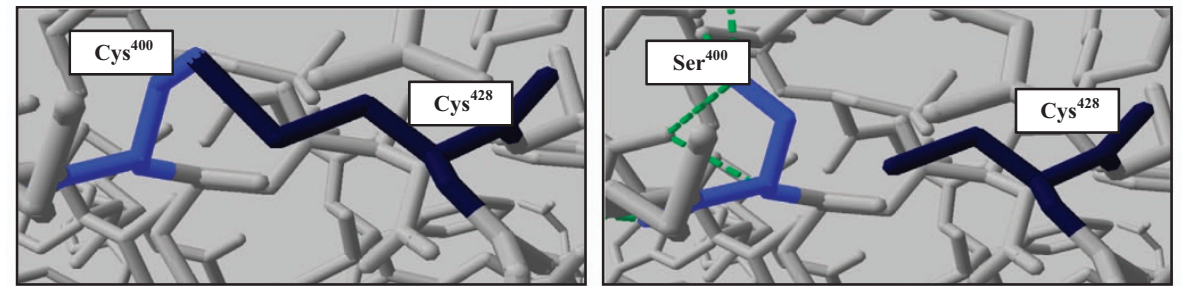

Figure 1. (a) Schematic diagram of the FVII structure features adopted by UniProtKB (P08709, FA7_HUMAN). A focus of the area of the FVII structure including Cys400 and Cys428 is shown. Disulfide bridge between Cys400 and Cys428 is highlighted and labeled. (b) Multiple alignment of FVII protein sequences generated by MUSCLE version 3.6 (using option: -maxiters 2) from HomoloGene automated system. The Cys400 residue (according to $H$. sapiens FVII sequence numbering) is shown in the narrow box. NP_062562.1: H. sapiens; XP_001149885.2: P. troglydytes; NP_001073605.1: M. mulatta; NP_001041498.1: C. lupus; NP_001029978.1: B. taurus; NP_034302.2: M. musculus; NP_690059.1: R. norvegicus; NP_989773.1: G. gallus; NP_001083027.1: D. rerio; NP_001083027.1: X. tropicalis. (c) Molecular modeling performed by SwissPdbViewer application. Visualizations of the disulfide bridge Cys400-Cys428 (on the left) in the Cys400-FVII structure (PDB code: 2C4F) and of the broken disulfide bridge Cys400-Cys428 (on the right) in the Ser400-FVII structure (PDB code: 2C4F) are shown.

c.-54G $>$ A (rs367732974) within the F7 $5^{\prime}$-upstream region and c.509G >A (rs375134790) within exon 6 and found to be responsible for the p.Arg170His missense change. To our knowledge, c.-54G > A and c.509G > A (p.Arg170His) have never been described in FVII-deficient individuals.

Molecular findings and bleeding symptoms in homozygous/ compound heterozygous patients

We identified 2 compound heterozygous and 1 homozygous patients with FVII deficiency (median of FVII 5.1\% (1.5-5.5)). No novel variants have been identified in this group. We identified one splicing-site variant and three variants responsible for a missense change. All three patients belonging to this group were symptomatic. Patient \#3 showed FVII activity of $5.1 \%$ and homozygosis for the previously reported c.641T>G variant, known to be responsible for the p.Val214Gly missense change (www.factorvii.org). The patient presented with a history of epistaxis. He was referred to our center before surgery for a pilonidal sinus cyst and, on this occasion, he was successfully managed using FVlla at a dose of $20 \mathrm{lU} / \mathrm{kg}$ immediately before surgery and every $6 \mathrm{~h}$ following the procedure. Both of the compound heterozygous patients were found to be carriers of the previously described c.1171 G>A variant, known to be responsible for the p.Gly391Ser missense change (www.factorvii.org). On the other allele, patient \#5 (FVII 5.5\%) and patient \#7 (FVII 1.5\%) showed previously reported variants: c.805+1G $>$ A and c.911C $>$ T. Patient \#5 arrived at the emergency room of our institute with a deep vein thrombosis and pulmonary embolism and presenting a thoracic hematoma, occurring after a fall from a stairway. He was treated with $30 \mu \mathrm{g} / \mathrm{kg}$ every $12 \mathrm{~h}$ and with enoxaparin $8000 \mathrm{IU}$ b.i. d. This patient tested negative for thrombophilia conditions. Patient \#7 (FVII 1.5\%) presented with a history of post-traumatic ecchymoses.

Molecular findings and bleeding symptoms in heterozygous patients

We identified 7 heterozygous patients with FVII deficiency (median of FVII $36.7 \%$ (16.5-51)). In this group, we identified two variants within F7 promoter sequences, one splicing-site variant and four variants responsible for a missense change. Four of seven patients were asymptomatic. One of the asymptomatic patients (patient \#10) was found to be a carrier of the undescribed polymorphic variant c.509G $>A$, which is responsible for the $p$. Arg170His missense change. The remaining 3 asymptomatic 
a

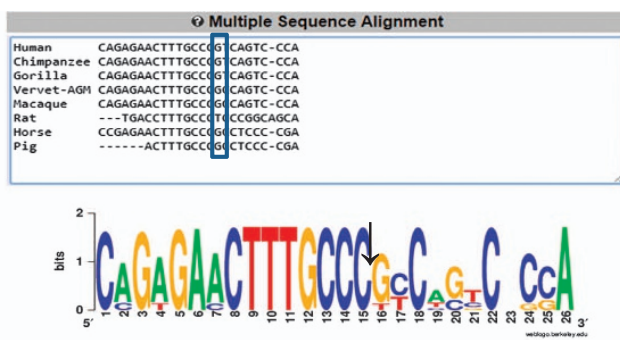

b
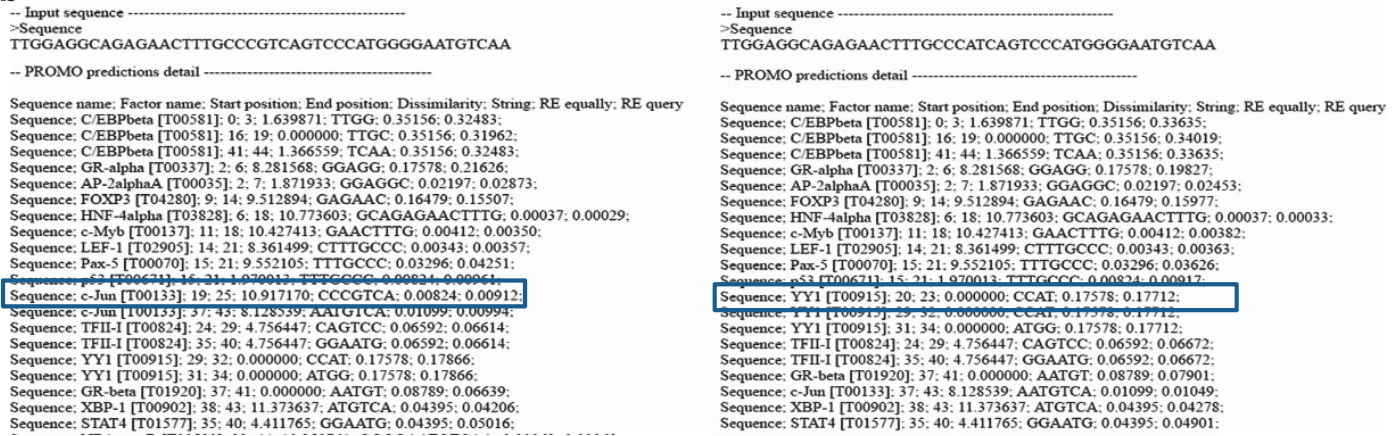

Figure 2. (a) Multiple alignment of $F 7$ sequences including the c.-54G nucleotide. Arrow indicates the c.-54G position. (b) PROMO software predictions of pattern of potential binding sites for TFs in F7 promoter sequences, comparing - 54G (left side) to - 54A (right side) alleles. The presence of $-54 \mathrm{~A}$ allele abolishes a binding site for c-Jun TF (within square brackets) and induces a novel binding site for the repressor YY1 TF (within square brackets).

patients were found to be carriers of previously reported variants (Table 1). We recorded a spontaneous epistaxis and episodes of rectal bleeding in 1 (patient \#2) of 7 symptomatic patients. Patient \#2 was found to be a carrier of the novel c.1199G >C allele variation, which is responsible for the p.Cys400Ser missense change. The Cys400 residue forms a disulfide bridge with Cys428 in the FVII structure (Figure 1a). ${ }^{22}$ Multiple alignments of FVII protein sequences show that Cys400 is a residue with high evolutionary conservation (Figure $1 \mathrm{~b}$ ). Furthermore, modeling of the FVII structure suggests that the presence of a serine residue at the 400 position instead of a cysteine in the FVII protein could lead to the breaking of the Cys400-Cys428 disulfide bridge (Figure 1c). Polyphen-2 (Polyphen-2: score of 1.00 ; sensitivity 0.00 , specificity 1.00) and SIFT (SIFT: score of 0.00 ; Median Conservation Information 2.57) predictions show that the p.Cys400Ser change could be classified as probably damaging and as deleterious, respectively. A bleeding diathesis was observed in the 2 patients with variants within F7 promoter sequences. Patient \#4 was found to be a carrier of the undescribed c.-54G $>$ A polymorphic variant. The patient was a 26-year-old woman (FVII: 39\%) with a history of episodes of menorrhagia successfully treated with $15 \mu \mathrm{g} / \mathrm{kg}$ recombinant FVIla every $12 \mathrm{~h}$ to fully control bleeding symptoms (she had partially responded to tranexamic acid). The c.-54G is close to a binding site (at nucleotides -67 to -56 ) for hepatocyte nuclear factor-4 (HNF-4) and to a major transcription start site at nucleotide $-51 .{ }^{23}$ Comparative genomics between human $\mathrm{F} 7$ and seven other eutherian mammals (Pan troglodytes, Gorilla gorilla, Chlorocebus sabaeus, Macaca mulatta, Rattus norvegicus, Equus caballus, and Sus scrofa) identified human c.-54G as a highly conserved allele, resulting in a nonalignable allele with Rattus norvegicus F7 sequences (Figure 2a). In silico predictions identified a potential novel putative binding site for transcriptional repressor protein YY1 and a potential loss of the c-Jun binding site induced by $c .-54 G>A$ (Figure 2b). The other patient, a carrier of the previously reported c.-30A $>\mathrm{C}$ variant, ${ }^{24}$ was a 10 -year-old girl (\#6; FVII: $36.7 \%$ ) who had experienced a perinatal intracranial hemorrhage (she was delivered at 32 weeks of gestation and weighed $1800 \mathrm{~g}$ ) and no other bleeding symptoms in her lifetime.

\section{DISCUSSION}

In our series, bleeding manifestations seemed to be quite heterogeneous, from epistaxis to vaginal bleeding and ecchymoses/hematoma, confirming the peculiarity of the deficiency of presenting no specific bleeding tendency. We observed only 1 severe hemorrhage (perinatal intracranial bleeding) in a heterozygous patient who experienced a preterm birth (born at 32 weeks of gestation) and carried the c.-30A $>C$ variant. Intracranial bleeding is commonly observed in preterm infants, and the pathogenesis is based on the interplay of multifactorial and complex mechanisms. ${ }^{25}$ Thus, it is plausible that the intracranial bleeding in patient \#6 occurred independently of the deficiency, or it could be that the deficiency accentuated the bleeding. We confirmed a poor correlation between FVII levels and bleeding phenotypes; bleeding manifestations were equally distributed among individuals with very low and slightly reduced FVII levels, as well as among homozygous/compound heterozygous and heterozygous patients. It was shown that heterozygous individuals can have bleeding manifestations, ${ }^{26-29}$ although other authors ${ }^{13}$ did not confirm this observation. In agreement with others, ${ }^{26-29}$ we describe a bleeding phenotype in three heterozygous patients carrying a novel missense change (p.Cys400Ser), an undescribed polymorphic variant (c.-54G $>A$ ) within the $F 7$ promoter, and one previously reported variant $(\mathrm{c} .-30 \mathrm{~A}>\mathrm{C})$ within the $F 7$ promoter. Subjects with the FVII deficiency and with a heterozygous genotype are usually asymptomatic. We recorded and observed bleeding symptoms in the heterozygous patients referred to our center. In the heterozygotes, we believe that the attributable risk for bleeding in FVII deficiency has to be evaluated case-by-case, addressing the contribution of further individual risk factors to the bleeding. The presence of bleeding symptoms in heterozygous subjects described in our series once more highlights the unclear relation between FVII levels and bleeding symptoms. 


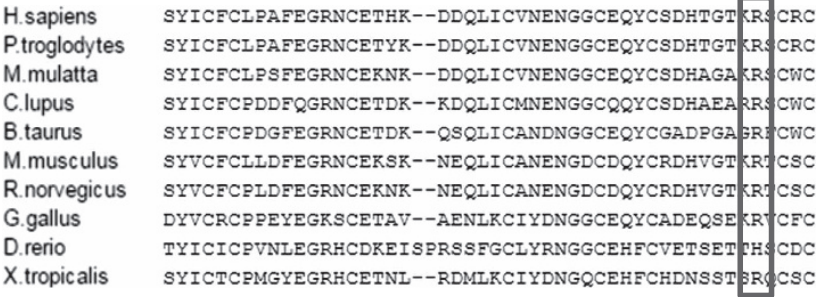

Figure 3. Multiple alignment of FVII protein sequences generated by MUSCLE version 3.6 (using option: -maxiters 2) from HomoloGene automated system. The Arg170 residue (according to $H$. sapiens FVII sequence numbering) is shown in the narrow box. NP_062562.1: $H$. sapiens; XP_001149885.2: $P$. troglydytes; NP_001073605.1: M. mulatta; NP_001041498.1: C. lupus; NP_001029978.1: B. taurus; NP_034302.2: M. musculus; NP_690059.1: R. norvegicus; NP_989773.1: G. gallus; NP_001083027.1: D. rerio; NP_001083027.1: X. tropicalis.

In silico predictions evidenced alterations of the pattern of transcription factor binding sites induced by the c.-54G $>A$ variant. Across-species alignment could suggest a crucial position related to the c.-54G allele, although the alignment approach of noncoding sequences could be influenced by specific sequence parameters, e.g., transcription factor binding sites or GC-rich regions. We believe that the c.-54G $>$ A substitution could have an impact on $F 7$ regulation since the $-54 G$ is close to a binding site (nucleotides -67 to -56 ) for hepatocyte nuclear factor-4 (HNF-4) and to a major transcription start site at nucleotide -51 . It could be that the presence of the A allele at position - 54 could interfere with the binding of $F 7$ regulatory sequences with transcription factors. To our knowledge, the c.-54G $>$ A has never been reported in individuals with FVII deficiency. Patient \#4 did not show other variants in F7 that could represent a genetic determinant of FVII levels (FVII: 39\%) except homozygosis for the well-known polymorphic variants (A2A2/QQ) of $F 7 .^{10}$ In the Exome Aggregation Consortium (ExAC), which includes analysis of 60706 individuals from more than 20 research studies, the c.-54G $>A$ is shown to have an allele frequency of 0.0001238 (with 1 allele identified in 23428 analyzed) and no A allele results identified in European populations. ${ }^{30}$ Therefore, the role of the c.-54G $>$ A in determining biochemical phenotype deserves further investigation.

The novel missense change p.Cys400Ser, which has been identified in a heterozygous patient with epistaxis and rectal bleeding episodes, involves a residue lying in the catalytic domain of FVII and leads to the disruption of the Cys400-Cys428 disulfide bridge. Higashi et al. $^{31}$ demonstrated that the Cys400-Cys428 disulfide bridge contributes to the amidolytic activity of FVII and facilitates active conformation of the factor. In keeping with this, multiple alignments of FVII sequences suggested that the Cys400 residue could be crucial for FVII structure, given high evolutionary conservation (Figure 2b). In addition, in silico predictions obtained by using Polyphen-2 and SIFT applications suggested a damaging effect of a Cys400Ser missense change on FVII structure/function. This missense change has been found to be associated with epistaxis and rectal bleeding episodes. However, we cannot exclude that rectal bleeding could be due to rectal lesions shown by patient \#2.

There are no case series on FVII deficiency describing patients carrying variant c.509G $>$ A (p.Arg170His). We found the p.Arg170His missense change in patient \#10, who showed no other variants in F7 except homozygosis for the well-known polymorphic variants (A2A2/QQ). The patient's sons showed FVII deficiency (24 and 31\%; data not reported in Table 1) and heterozygosis for the p.Arg $170 \mathrm{His}$ missense change and for the well-known F7 polymorphic variants (A1A2/RQ). The c.509G $>A$ shows a minor allele frequency (MAF) of $<0.01$ in dbSNP. In silico predictions revealed contrasting effects related to a p.Arg170His missense change (Polyphen-2: score 0.7 , sensitivity 0.86 , specificity 0.92, possible damaging; SIFT: score 0.56, median conservation information 2.57, tolerated). Multiple alignment of F7 sequences showed a high evolutionary conservation of Arg170 (Figure 3). However, the possible role of a p.Arg170His missense change as a common missense variant and as a genetic determinant of FVII levels needs to be clarified.

In agreement with other studies on patients with FVII deficiency from southern Italy, we observed that the p.Gly391Ser missense change was the most frequently identified variant in patients studied in our center. Indeed, Etro et $a l^{32}$ suggested screening the subjects from southern Italy with FVII deficiency for this variant, since they observed that the p.Gly391Ser shows a high frequency in subjects from this geographical area. Most of the homozygous/ compound heterozygous patients reported in the paper by Etro et al. were asymptomatic. At variance with these clinical data, we observed bleeding symptoms in both the compound heterozygotes carrying p.Gly391Ser when the variant was found associated with the presence of the $c .805+1 \mathrm{G}>\mathrm{A}$ variant or the p.Ala304Val missense change. Thus, the role of the p.Gly391Ser missense change in determining bleeding in FVII-deficient Italian patients remains to be further investigated.

Our patients showed bleeding symptoms mainly in presence of triggers such as trauma and stairway falls or on the occasion of heavy menstrual periods and as complications of a preterm birth. Furthermore, 1 patient (patient \#5) with severe FVII deficiency was asymptomatic until the occurrence of a trauma; surprisingly, after a fall he was diagnosed with pulmonary embolism in absence of a thrombophilia state. Association between FVII deficiency and venous thromboembolism (VTE) has been previously described. ${ }^{33,34}$ In patient \#5, we hypothesized that a traumatic event could trigger both bleeding and VTE.

In conclusion, this paper adds novel insights into molecular and clinical aspects of FVII deficiency. We observed spontaneous bleeding symptoms (epistaxis) among heterozygous (patient \#2) and homozygous (patient \#3) patients, as well as bleeding symptoms after hemostatic challenges (trauma, stairway fall, rectal lesions) or on the occasion of heavy menstrual periods and as complications of a preterm birth among compound heterozygous (patients \#5 and \#7) and heterozygous (patients \#2, \#4 and \#6) patients. Our findings further suggest that, independent of FVII levels and of the variants and the genotype affecting FVII levels, environmental factors, e.g., trauma, could heavily influence the clinical phenotype of FVII-deficient patients.

\section{ACKNOWLEDGEMENTS}

GLT, MM, and EG created the study concept and design; GF, EC and DC performed molecular investigations; LF performed plasma-based assays; GLT, MI and MM performed in silico predictions; GLT wrote the paper; and MM and EG reviewed and revised the paper.

\section{COMPETING INTERESTS}

The authors declare no conflict of interest.

\section{PUBLISHER'S NOTE}

Springer Nature remains neutral with regard to jurisdictional claims in published maps and institutional affiliations.

\section{REFERENCES}

1 Hoffman M, Monroe DM 3rd. A cell-based model of haemostasis. Thromb Haemost 2001; 85: 958-965.

2 Monroe DM, Hoffman M, Roberts HR. Transmission of a procoagulant signal from tissue factor-bearing cell to platelets. Blood Coagul Fibrinolysis 1996; 7: 459-464. 
3 Hoffman M, Monroe DM. Coagulation 2006: a modern view of hemostasis. Hematol Oncol Clin North Am 2007; 21: 1-11.

4 Broze GJ Jr, Majerus PW. Purification and properties of human coagulation factor VII. J Biol Chem 1980; 255: 1242-1247.

5 Greenberg DL, Davie EW. The blood coagulation factors-their complementary DNAs, genes, and expression. In: Colman RW, Marder VJ, Clowes AW, George JN, Goldhaber SZ (eds.) Hemostasis and Thrombosis: Basic Principles and Clinical Practice, 5th Chapter 3. Lippincott Williams and Wilkins: New York, 2006, 21-57.

6 O'Hara PJ, Grant FJ, Haldeman BA, Gray CL, Insley MY, Hagen FS et al. Nucleotide sequence of the gene coding for human factor VII, a vitamin K-dependent protein participating in blood coagulation. Proc Natl Acad Sci USA 1987; 84: 5158-5162.

7 Perry DJ. Factor VII deficiency. Br J Haematol 2002; 18: 689-700.

8 Sevenet PO, Kaczor DA, Depasse F. Factor VII deficiency. Clin Appl Thromb Hemost 2016; 23: 703-710.

9 Girolami A, Cosi E, Santarossa C, Ferrari S, Luigia Randi M. The story of serum prothrombin conversion accelerator, proconvertin, stable factor, cothromboplastin, prothrombin accelerator or autoprothrombin I, and their subsequent merging into factor VII. Semin Thromb Hemost 2015; 41: 366-373.

10 Bernardi F, Marchetti G, Pinotti M, Arcieri P, Baroncini C, Papacchini M et al. Factor VII gene polymorphisms contribute about one third of the factor VII level variation in plasma. Arterioscler Thromb Vasc Biol 1996; 16: 72-76.

11 Giansily-Blaizot M, Schved JF. Potential predictors of bleeding risk in inherited factor VII deficiency. Clinical, biological and molecular criteria. Thromb Haemost 2005; 94: 901-906.

12 Triplett DA, Brandt JT, Batard MA, Dixon JL, Fair DS. Hereditary factor VII deficiency: heterogeneity defined by combined functional and immunochemical analysis. Blood 1985; 66: 1284-1287.

13 Girolami A, Cosi E, Ferrari S, Girolami B, Lombardi AM. Bleeding manifestations in heterozygotes with congenital FVII deficiency: a comparison with unaffected family members during a long observation period. Hematology 2017; 22: 375-379.

14 Margaglione M, Brancaccio V, Giuliani N, D'Andrea G, Cappucci G, lannaccone L et al. Increased risk for venous thrombosis in carriers of the prothrombin G-->A20210 gene variant. Ann Intern Med 1998; 129: 89-93.

15 Messeguer X, Escudero R, Farré $D$, Núñez $O$, Martínez J, Albà MM. PROMO: detection of known transcription regulatory elements using species-tailored searches. Bioinformatics 2002; 18: 333-334.

16 Flicek P, Amode MR, Barrell D, Beal K, Billis K, Brent S et al. Ensembl 2014. Nucleic Acids Res 2014; 42(Database issue): D749-D755.

17 Crooks GE, Hon G, Chandonia JM, Brenner SE. WebLogo: a sequence logo generator. Genome Res 2004; 14: 1188-1190.

18 Edgar RC. MUSCLE: multiple sequence alignment with high accuracy and high throughput. Nucleic Acids Res 2004; 32: 1792-1797.

19 Kumar P, Henikoff S, Ng PC. Predicting the effects of coding non-synonymous variants on protein function using the SIFT algorithm. Nat Protoc 2009; 4: 1073-1108.

20 Adzhubei I, Jordan DM, Sunyaev SR. Predicting functional effect of human missense mutations using PolyPhen-2. Curr Protoc Hum Genet 2013; Chapter 7: Unit 7.20.

21 Guex N, Peitsch MC. Swiss-Model and the Swiss-Pdb Viewer: an environment for comparative protein modeling. Electrophoresis 1997; 18: 2714-2723.
22 Takamiya O, Seta M, Tanaka K, Ishida F. Human factor VII deficiency caused by S339C mutation lacated adjacent to the specificity pocket of the catalytic domain. Clin Lab Haematol 2002; 24: 233-238.

23 Pollak ES, Hung HL, Godin W, Overton GC, High KA. Functional characterization of the human factor VII 5'-flanking region. J Biol Chem 1996; 271: 1738-1747.

24 Millar DS, Kemball-Cook G, McVey JH, Tuddenham EG, Mumford AD, Attock GB et al. Molecular analysis of the genotype-phenotype relationship in factor VII deficiency. Hum Genet 2000; 107: 327-342.

25 McCrea HJ, Ment LR. The diagnosis, management, and postnatal prevention of intraventricular hemorrhage in the preterm neonate. Clin Perinatol 2008; 35: 777-792.

26 Herrmann FH, Wulff K, Auerswald G, Schulman S, Astermark J, Batorova A et al. Greifswald Factor FVII Deficiency Study Group. Factor VII deficiency: clinical manifestation of 717 subjects from Europe and Latin America with mutations in the factor 7 gene. Haemophilia 2009; 15: 267-280.

27 Mahmoodi M, Peyvandi F, Afrasiabi A, Ghaffarpasand F, Karimi M. Bleeding symptoms in heterozygous carriers of inherited coagulation disorders in southern Iran. Blood Coagul Fibrinolysis 2011; 22: 396-401.

28 Fromovich-Amit Y, Zivelin A, Rosenberg N, Tamary H, Landau M, Seligsohn U. Characterization of mutations causing factor VII deficiency in 61 unrelated Israeli patients. J Thromb Haemost 2004; 2: 1774-1781.

29 Acharya SS, Coughlin A, Dimichele DM. North American Rare Bleeding Disorder Study Group. Rare Bleeding Disorder Registry: deficiencies of factors II, V, VII, X, XIII, fibrinogen and dysfibrinogenemias. J Thromb Haemost 2004; 2: 248-256.

30 Lek M, Karczewski KJ, Minikel EV, Samocha KE, Banks E, Fennell T et al. Exome Aggregation Consortium. Analysis of protein-coding genetic variation in 60,706 humans. Nature 2016; 536: 285-291.

31 Higashi S, Matsumoto N, Iwanaga S. Conformation of factor Vlla stabilized by a labile disulfide bond (Cys-310-Cys-329) in the protease domain is essential for interaction with tissue factor. J Biol Chem 1997; 272: 25724-25730.

32 Etro D, Pinotti M, Wulff K, Marchetti G, Rocino A, Herrmann FH et al. The Gly331Ser mutation in factor VII in Europe and the Middle East. Haematologica 2003; 12: 1434-1436.

33 Mariani G, Herrmann FH, Schulman S, Batorova A, Wulff K, Etro D et al. International Factor VII Deficiency Study Group. Thrombosis in inherited factor VII deficiency. J Thromb Haemos 2003; 1: 2153-2158.

34 Girolami A, Berti de Marinis G, Vettore S, Girolami B. Congenital FVII deficiency and pulmonary embolism: a critical appraisal of all reported cases. Clin Appl Thromb Hemost 2013; 19: 55-59.

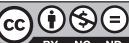

This work is licensed under a Creative Commons AttributionNonCommercial-NoDerivs 4.0 International License. The images or other third party material in this article are included in the article's Creative Commons license, unless indicated otherwise in the credit line; if the material is not included under the Creative Commons license, users will need to obtain permission from the license holder to reproduce the material. To view a copy of this license, visit http:// creativecommons.org/licenses/by-nc-nd/4.0/

(c) The Author(s) 2017 